\title{
Metric Characteristics of Tests Assessing Speed and Agility in Youth Soccer Players
}

\author{
Zeljko Kovacevic' ${ }^{1}$, Frane Zuvela ${ }^{2}$ and Goran Kuvacic ${ }^{2}$ \\ 'University of Split, Department of Health Studies, Split, Croatia, ${ }^{2}$ University of Split, Faculty of Kinesiology, Split, Croatia
}

\section{Abstract}

The main aim of this study was to validate the measuring instruments for assessing sprinting speed and specific agility in youth soccer players. The study was conducted on a sample of 32 soccer players in younger age categories. The sample of variables included three tests assessing sprinting speed (5-meter sprint from a standing start, 20-meter sprint from a standing start, and flying 15-meter sprint), and three tests assessing specific agility (slalom run with a ball, zig-zag run with a ball, and 20-meter sprint with a ball). Metric characteristics of tests were determined by calculating indicators of reliability, homogeneity, sensitivity, and factor and pragmatic validity. The values of indicators of metric characteristic showed that the applied motor tests had high level of reliability, good homogeneity, normal distribution of data, and acceptable values of factor and pragmatic validity. As a recommendation for future scientific and/or expert activities which would include assessment of speed and specific agility in young soccer players, investigations should definitely include 20-meter sprint from a standing start and slalom run with a ball.
\end{abstract}

Key words: metric characteristics, soccer players, agility, speed

\section{Introduction}

One of the main reasons for widespread popularity of soccer today is in its full "exploitation" of athlete's conditional and technical-tactical performances. One can be successful in soccer, in either offense or defense, only if the fundamental, i.e., basic functional and motor abilities are at high level. Whether it be a top professional or recreational athlete, the overall volume of activity during a soccer match or training requires from an athlete the maximum activation of power, balance, flexibility, coordination, precision, endurance, and speed and specific agility.

Soccer is dominated by frequent and rapid changes of movement direction, sprints, jumps, sudden stops (Váczi, Tollár, Meszler, Juhász, \& Karsai, 2013), so it can be assumed that motor dimensions responsible for performance of these movements greatly differentiate elite soccer players from average players or amateurs. Ball possession, total shots on goal and assists contribute most to the final team placement in a competition (Lago-Peñas, Lago-Ballesteros, Dellal, \& Gómez, 2010) There have been some studies (Buchheit, Mendez-Villanueva, Delhomel,
Brughelli, \& Ahmaidi, 2010; Mujika, Santisteban, Impellizzeri, \& Castagna, 2009) that suggest that abilities such as speed, agility, and explosive power are more determinant of success than others. By reaching puberty, which is marked by significant release of hormones, increase of height and body mass, and most importantly, increase of muscle mass, biologically more mature individuals are enabled to demonstrate greater force per unit of time and therefore achieve better results in sprint tests (De Matos et al., 2013; Le Gall, Carling, Williams, \& Reilly, 2010; Malina, Eisenmann, Cumming, Ribeiro, \& Aroso, 2004), repeated sprint (Mendez-Villanueva et al., 2011), explosive power (Chuman, Hoshikawa, \& Lida, 2009; Figueiredo, Gonçalves, Coelho E Silva, \& Malina, 2009), and agility (De Matos et al., 2013; Figueiredo et al., 2009).

Speed and specific agility are mutually correlated and, as such, represent one of the main factors of success in soccer. Speed as a movement ability is manifested as an activity in which a person attempts to cover a given distance from start to finish in the shortest time possible. Speed as a motor ability is very im-

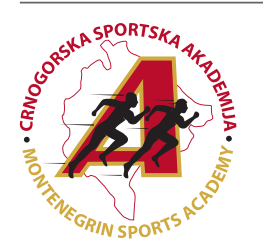

Correspondence:

G. Kuvacic

University of Split, Faculty of Kinesiology, Teslina 6, Split, Croatia

e-mail: gorkuv@kist.hr 
portant in all sports, thus in soccer as well. Even though speed is greatly ,innate“, its performance may be somewhat influenced primarily by a process of motor learning of those performance factors which directly determine the quality of technique of the starting position, start, starting acceleration, gaining maximum speed, as well as running when reaching the finish line (stride length and frequency,knowledge of the starting skill, etc.).

Agility is coordination in reaction to what is happening on the field and performance without delay (Gatz, 2009). As in any other sport, differences in agility between individuals in soccer are noticeable in activities which are manifested during performance of the first stride, as well as in the ability and efficiency of stopping and starting to moveagain and changing movement direction. Generally, both abilities include movement in the sense of rapid forward motion, only that agility also includes the change of movement direction. Measurement/testing of speed and agility can help and identify athlete's weaknesses within the sport or the quality of performance of a certain task (Harman \& Garhammer, 2008). Assessment of speed and agility (specific coordination) in controlled conditions with tests which are similar to the demands of play in the sport itself, is very efficient in helping the programming of training, with the aim of higher performance quality of athletes in competitive conditions (Miller, 2012).

Therefore, the main aim of this study is to validate the measuring instruments for assessing speed and specific agility of youth soccer players.

\section{Methods}

The study was conducted on a sample of 32 young soccer players in two age categories. The first group of soccer players $(\mathrm{N}=20)$ included children whose chronological age was $10 \pm 1.0$ years (height: $142.4 \pm 5.4 \mathrm{~cm}$; weight: $37.1 \pm 5.8 \mathrm{~kg}$; body mass index: $18.2 \pm 1.8$; body fat $\%$ : $15.9 \pm 6.1)$, whereas the second group of young soccer players $(\mathrm{N}=12)$ included children aged $11 \pm 0.6$ years (height: $145.6 \pm 5.1 \mathrm{~cm}$; weight: $37.7 \pm 4.0$; body mass index: $17.9 \pm 2.0$; body fat $\%: 14.6 \pm 3.4$ ).

To identify subjects with a medical contraindication to performing applied tests, subjects completed medical history questionnaires.Inclusion criteria to participate in this study were: i) at least one year of training experience, ii) having a valid sport medical certification, and iii) being healthy (i.e. absence of musculoskeletal or joint injuries, cardiovascular disease, and illness,). Subjects did not eat for 2 hours before testing to reduce any possible interference on the experiment. Written consent for participation in this study was obtained from the subject's parents/guardians after being thoroughly informed about the purpose, benefits, and potential risks of this study.The Ethical Committee of the Faculty of Kinesiology, University of Split verified that this investigation complied with all ethical standards for scientific investigations involving human subjects.

\section{Testing procedures}

To eliminate any influence of circadian variation, each participant completed all trials in the same time period of the testing day and under the same climate conditions (8-10 p.m., $24.6 \pm 0.1^{\circ} \mathrm{C}$ temperature and $30.3 \pm 1.9 \%$ relative humidity). Twenty-four hours before the testing protocol subjects weren't under heavy training routines. In this way, no interference of fatigue on the results was possible.

Prior to testing, anthropometric characteristics (standing height, body weight, body mass index, and body fat\%) were car- ried out. Standing height was measured with Harpenden Portable Stadiometer 603 VR (Holtain LTD, Crosswell, UK). Body mass index and body fat $\%$ were calculated using Tanita diagnostic scale BC 418 (Tokyo, Japan). After anthropometric measurements, subjects performed tests assessing speed and specific agility. Witty Timing System's (Microgate, Bolzano, Italy) time gate was used to record time. All tests were performed on a flat surface, i.e., artificial grass. All subjects had soccer boots and the ball used in all tests was Adidas Jabulani $69.0 \pm 0.2 \mathrm{~cm}$ in circumference and $440 \pm 0.3 \mathrm{~g}$ in mass. Testing protocol included quality warm-up of 25 minutes because this investigation required maximal muscle activation during a short period of time. Warm-up included sequences of 15 minutes for jogging with and without the ball followed by 10 minutes of dynamic stretching especially of leg and abdominal muscles.

Speed was assessed by the following tests: MT5M-5-meter sprint from a standing start, MT20M-20-meter sprint from a standing start, MT15ML-15-meter sprint from a flying start. In tests assessing sprinting speed, subject stood in a standing start position $1 \mathrm{~m}$ behind the start line where the first pair of photocells was placed.Depending on the test, the second pair of photocells was placed at 5, 15 or 20 meters. A subject started to move arbitrarily, and his task was to run the distance between the photocells at maximum speed.

To assess specific agility following tests were applied:M20 L-20-meter sprint from a standing start with a ball, MSLA Lslalom run with a ball, MZI-ZA L-zig-zag run with a ball.20-m run with a ball $(\mathrm{M} 20 \mathrm{~L})$ - the subject stands in a medium start with a ball $1 \mathrm{~m}$ behind the start line, where the first pair of photocells is placed. The second pair of photocells is placed at $20 \mathrm{~m}$. The subject starts to run arbitrarily, and his task is to cover the distance between the photocells with maximum speed by dribbling the ball and he must touch it at least 4 times - at least once in the first $3 \mathrm{~m}$.Slalom test (MSLAL)-the test is performed on a flat surface, i.e., artificial grass. The subject stands in a medium start position with a ball at the start line, straight in front of him at $1 \mathrm{~m}$ there are 6 slalom poles $2 \mathrm{~m}$ apart, and the subject must dribble the ball between those poles at maximum speed forward and back. The subject starts arbitrarily, and time is measured from the moment he starts moving until he crosses the start/finish line with the ball.Zig - zag test (MZI-ZA L)the test is performed on a flat surface, i.e., artificial grass. The subject stands in a medium start position with a ball at the start line. The zig-zag test is constructed as a $16 \mathrm{x} 10$-yard $(4.85 \mathrm{~m} \mathrm{x}$ $3 \mathrm{~m}$ ) rectangle, with a cone right in the centre. The subject's task is to dribble the ball with maximum speed around the cones after the start he goes around the central cone, then around the two side cones, again around the central cone, and around the final cone towards the start/finish line. Each test is repeated 3 times with pause adequate for recovery.

\section{Statistical analysis}

In line with the main aim of the study-validation of measuring instruments for assessing sprinting speed and specific agility the following methods of data analysis were used: for sensitivity of applied tests, descriptive statistical parametersarithmetic mean (AM), standard deviation (SD), minimum (MIN) and maximum (MAX) result, measures of asymmetry (SKE) and peakedness (KURT) of the probability distribution, Kolmogorov-Smirnov test of normality of distribution (K-S) were calculated. For reliability of applied tests Inter-item correlation (IIr) and Cronbach's alpha ( $\alpha$ ) were calculated. Univari- 
ate analysis of variance (F, p)-differences between results of each repetition for each variable was used to calculate homogeneity. To determine factor validity of tests assessing sprinting speed and specific agility, the intercorrelation matrix was transformed into a principal component matrix. By doing so, projections of variables on the first principal component (Guttman-Kaiser criterion) were obtained for, tests assessing sprinting speed and specific agility. By applying an independent samples t-test to assess differences between two groups of subjects, pragmatic validity was determined. Statistical significance was designated at $\mathrm{p}<0.05$. Statistical analyses were carried out using Statistica software version 13.2 (Dell Inc., Round Rock, TX, USA).

\section{Results}

In line with the main aim of the study, the results of metric characteristics of tests assessing sprinting speed and specific agility in youth soccer players are presented below.

\section{Metric characteristics of tests assessing speed}

Indicators of reliability of tests assessing speed showed rel- atively high correlation between the measured items in all the analysed motor tests (Table 1). The values of reliability coefficient of „inter-item correlation“ (IIr) ranged from moderately high correlation in the 5 -meter sprint from a standing start (MT5M; IIr=0.68) to high correlation in the 20-meter sprint from a standing start (MT20M; IIr=0.97). The results of the Cronbach's alpha coefficient of reliability ( $\alpha$ ) also indicated a relatively high correlation between the measured items (V1-V3) in all the analysed tests assessing sprinting speed. The values of coefficient of reliability $(\alpha)$ ranged from high correlation value in the MT5M test ( $\alpha=0.84)$ to very high correlation value in the MT20M test $(\alpha=0.97)$. Examination of indicators of the metric characteristic (Table 1) which generally indicates to what extent the subjects' result in the analysed items (V1-V3) depends on the same object of measurement, showed that unambiguous inference (homogenous test) is possible in all the applied tests assessing sprinting speed. Thus, as end result in all the tests: 5-meter sprint from a standing start (MT5M; $\mathrm{p}=0.331$ ), 20-meter sprint from a standing start (MT20M; $\mathrm{p}=0.001)$ and flying 15 -meter sprint (M15ML; $\mathrm{p}=0.41$ ), mean value was used.

\begin{tabular}{cccccccccc}
\multicolumn{8}{c}{ Table 1. Results of reliability and homogeneity of variables assessing sprinting speed } \\
\hline Variables & V 1 & V 2 & V 3 & II r & a & AM \pm SD & F-test & p \\
\hline \multirow{2}{*}{ MT5M (sec) } & 1.00 & 0.58 & 0.79 & & & $1.21 \pm 0.73$ & & \\
& 0.58 & 1.00 & 0.66 & 0.68 & 0.84 & $1.19 \pm 0.49$ & 1.10 & 0.331 \\
& 0.79 & 0.66 & 1.00 & & & $1.21 \pm 0.80$ & & \\
MT20M & 1.00 & 0.92 & 0.92 & & & $3.74 \pm 1.54$ & & \\
(sec) & 0.92 & 1.00 & 0.97 & 0.94 & 0.97 & $3.74 \pm 1.64$ & 4.29 & 0.170 \\
& 0.92 & 0.97 & 1.00 & & & $3.77 \pm 1.84$ & & \\
M15ML & 1.00 & 0.79 & 0.90 & & & $2.53 \pm 0.10$ & & \\
(sec) & 0.79 & 1.00 & 0.92 & 0.88 & 0.94 & $2.54 \pm 0.13$ & 2.31 & 0.106 \\
& 0.90 & 0.92 & 1.00 & & & $2.55 \pm 0.12$ & & \\
\hline
\end{tabular}

Legend: V1-V3-intercorrelation of items; IIr-inter-item correlation; a-Cronbach's alpha coefficient; AMarithmetic mean; SD-standarddeviation; $F$ test-analysis of variance; $\mathrm{p}$-significance, MT5M-5-meter sprint from a standing start, MT20M-20-meter sprint from a standing start, M15ML-flying 15-meter sprint.

By examining the values of sensitivity indicators in the analysed variables (Table 2 ), it can be said that all the tests (MT5M: $=0.16$; MT20M: D $\max =0.15$; M15ML: D $\max =0.16$ ) had distribution of results which does not deviate significantly from normal Gaussian distribution. Thus, as the analysed tests are successful in differentiating subjects regarding the object of measurement, all the tests are suitable for further statistical analyses planned in this study. The coefficient of asymmetry (SKE) shows if the majority of results of some test skew to the zone of maximum or minimum values. However, before analysing this indicator, it should be noted that the variable 5-meter sprint from a standing start (MT5M) is inversely scaled (a higher value of the test means a weaker result and vice versa), so this should be taken into consideration during interpretation. By examining the obtained values of coefficient of asymmetry, it can be said that there was minimum asymmetry towards higher results of the test in the variable 5-meter sprint from a standing start (MT5M). Moreover, by examining the measure of peakedness (KURT), it can be seen that it was somewhat more pronounced in the variable 5-meter sprint from a standing start (MT5M). The obtained results indicate somewhat higher clustering of results around the mean.

Table 2. Results of sensitivity of variables for assessing sprinting speed

\begin{tabular}{lccccccc}
\hline \multicolumn{1}{c}{ Variables } & AM & SD & MIN & MAX & SKE & KUR & D max \\
\hline MT5M (sec) & 1.21 & 0.06 & 1.10 & 1.44 & 1.70 & 6.22 & 0.16 \\
MT20M (sec) & 3.75 & 0.17 & 3.43 & 4.22 & 0.75 & 0.63 & 0.15 \\
M15ML (sec) & 2.55 & 0.11 & 2.33 & 2.78 & 0.55 & -0.57 & 0.16 \\
\hline
\end{tabular}

Legend: AM-arithmetic mean, SD-standard deviation, MIN-minimum result, MAX-maximum result, SKE-measure of asymmetry, KURT-measure of peakedness, K-S-Kolmogorov Smirnov test of normality of distribution, $d=0.246$ for $\mathrm{N}=32(\mathrm{p}<0.05), \mathrm{MT} 5 \mathrm{M}-5$ - meter sprint from a standing start, MT20M-20-meter sprint from a standing start, M15ML-flying 15-meter sprint.

In the overall sample, factor analysis in the space of three manifest motor variables analysed a single dimension, i.e., factor, which accounts for $88 \%$ of the variance in the system (Table 3). The highest correlation with the object of measurement, i.e., the highest validity was found in the 20-meter sprint from a standing start (MT20M: 0.98) test. Tests assessing sprinting speed which are ranked in such a way should not be treated as the best, weak and the weakest tests, but rather as very good tests for assessing sprinting speed, and among these very good tests, the MT20M is the best. 
Table 3. Factor analysis of variables assessing sprinting speed

\begin{tabular}{cc}
\hline Variables & F1 \\
\hline MT5M & 0.90 \\
MT20M & 0.98 \\
M15ML & 0.93 \\
Lambda & 2.64 \\
Variance $\%$ & 88.23
\end{tabular}

Legend: Lambda-eigenvalue; Variance \%-percentage of variance explained by latent dimension, MT5M-5- meter sprint from a standing start, MT20M-20-meter sprint from a standing start, M15ML-flying 15-meter sprint.

The results of differences determined by an independent samples t-test are presented in Table 4 . The results of the independent samples t-test indicate there was a statistically significant difference between two groups of soccer players only in the variable 5 -meter sprint ( $\mathrm{t}-\mathrm{value}=-2.56 ; \mathrm{p}=0.02$ ). By examining arithmetic means of the two groups of subjects, it can be concluded that soccer players in the first group (players aged 11 years) had better results.

Table 4. Differences between two groups of soccer players in tests assessing sprinting speed

\begin{tabular}{ccccc}
\hline Variable & $\begin{array}{c}\text { Group 1 } \\
(\mathbf{N}=\mathbf{2 0}) \\
\mathbf{A M} \pm \text { SD }\end{array}$ & $\begin{array}{c}\text { Group 2 } \\
(\mathbf{N}=12) \\
\mathbf{A M} \pm \text { SD }\end{array}$ & t-value & $\mathbf{p}$ \\
\hline MT5M & $1.15 \pm 0.04$ & $1.19 \pm 0.04$ & -2.56 & 0.02 \\
MT20M & $3.66 \pm 0.11$ & $3.75 \pm 0.14$ & -1.63 & 0.12 \\
M15ML & $2.49 \pm 0.24$ & $2.53 \pm 0.10$ & -1.21 & 0.24 \\
\hline
\end{tabular}

Legend: $t$-test-independent samples t-test, $p$-level of significance, MT5M5-meter sprint from a standing start, MT20M-20-meter sprint from a standing start, M15ML-fl ying 15-meter sprint.

Metric characteristics of tests assessing specific agility in youth soccer players

By examining coefficients of reliability of tests assessing specific agility (Table 5), it can be said that the values of inter-item correlation were relatively satisfactory. The most reliable tests for assessing the analysed ability were the zig-zag run with a ball (MZI-ZA L: IIr $=0.65 ; \alpha=0.83$ ) and the 20 -meter sprint with a ball (M20 L: IIr=0.6; $\alpha=0.85)$ test. On the other hand, somewhat lower accuracy of measurement was noted in the slalom run with a ball test (MSLA L: $\operatorname{IIr}=0.52 ; \alpha=0.75$ ). Results of homogeneity (F-test and the associated level of sig- nificance-p) indicate there were certain differences in values of indicators of this metric characteristic in tests assessing specific agility. Unambiguous inference (homogenous test) is possible in slalom run with a ball (MSLA: $\mathrm{p}=0.324)$ and zig-zag run with a ball test (MZI-ZA L: $\mathrm{p}=0.149$ ), whereas unambiguous inference (heterogenous tests) is not possible in the third test 20-meter sprint from a standing start with a ball $(\mathrm{M} 20 \mathrm{~L}$ : $\mathrm{p}=0.007)$. Therefore, as end result in the slalom run with a ball and zig-zag run with a ball test, mean value of the three measured items was used, whereas in the 20-meter sprint with a ball the best result was used.

Table 5. Results of reliability and homogeneity of variables assessing specific agility

\begin{tabular}{ccccccccc}
\hline Variables & V 1 & V 2 & V 3 & II $\mathbf{~}$ & $\mathbf{a}$ & $\mathbf{A M} \pm$ SD & F-test & $\mathbf{p}$ \\
\hline M20 L & 1.00 & 0.62 & 0.59 & & & $4.33 \pm 0.23$ & & \\
(sec) & 0.62 & 1.00 & 0.76 & 0.66 & 0.85 & $4.24 \pm 0.23$ & 5.33 & 0.007 \\
& 0.59 & 0.76 & 1.00 & & & $4.22 \pm 0.23$ & & \\
MSLA L & 1.00 & 0.55 & 0.46 & & & $12.58 \pm 1.57$ & & \\
(sec) & 0.55 & 1.00 & 0.55 & 0.52 & 0.75 & $12.39 \pm 1.50$ & 1.14 & 0.324 \\
& 0.46 & 0.55 & 1.00 & & & $12.20 \pm 1.09$ & & \\
MZI-ZA L & 1.00 & 0.82 & 0.49 & & & $9.53 \pm 0.66$ & & \\
(sec) & 0.82 & 1.00 & 0.59 & 0.65 & 0.83 & $9.70 \pm 0.75$ & 4.17 & 0.149 \\
& 0.49 & 0.59 & 1.00 & & & $9.84 \pm 0.75$ & & \\
\hline
\end{tabular}

Legend: V1-V3-inter-refereecorrelation; IIr-inter-item correlation; a-Cronbach's alpha coefficient; AMarithmetic mean; SD-standard deviation; $F$ test-analysis of variance; $\mathrm{p}$-level of significance, M20 L-20-meter sprint from a standing start with a ball, MSLA L-slalom run with a ball, MZI-ZA L-zig-zag run with a ball.

The results of sensitivity of tests (Table 6) showed that normal distribution of data existed in all the tests assessing specific agility. This was confirmed by the values of normality of distribution (D max), which did not deviate from relative cumulative Gaussian distribution (M20 L: D max=0.08; MSLA L: D max=0.13; MZI-ZA L: D $\max =0.12$ ). 
Table 6. Results of sensitivity of variables assessing specific agility

\begin{tabular}{cccccccc}
\hline Variables & AM & SD & MIN & MAX & SKE & KUR & D max \\
\hline M20 L (sec) & 4.16 & 0.19 & 3.86 & 4.63 & 0.38 & -0.28 & 0.08 \\
MSLA L (sec) & 12.39 & 1.15 & 10.30 & 15.37 & 0.73 & 0.83 & 0.13 \\
MZI-ZA L (sec) & 9.70 & 0.63 & 8.59 & 10.68 & -0.05 & -1.26 & 0.12 \\
\hline
\end{tabular}

Legend: AM-arithmetic mean, SD-standard deviation, MIN-minimum result, MAX-maximum result, SKE-measure of asymmetry, KURT-measure of peakedness, K-S-Kolmogorov Smirnov test of normality of distribution, $d=0.246$ for $N=32(p<0.05), M 20 L-20$-meter sprint from a standing start with a ball, MSLA Lslalom run with a ball, MZI-ZA L-zig-zag run with a ball.

Results of factor analysis of variables assessing specific agility are presented in Table 7. By factor analysis applied on a sample of youth soccer players, out of the 3 manifest variables assessing specific agility, a single latent dimension was isolated, accounting for $75.08 \%$ of total variability of the sys- tem. The obtained dimension was defined by high projection of the slalom run with a ball (MSLA L: 0.91) test, which is followed positively by good projection of the zig-zag run with a ball (MZI-ZA L: 0.89) and 20-meter sprint from a standing start with a ball (M20 L: 0.78) test.

Table 7. Factor analysis of variables assessing specific agility

\begin{tabular}{cc}
\hline Variables & F1 \\
\hline M20 L & 0.78 \\
MSLA L & 0.91 \\
MZI-ZA L & 0.89 \\
Lambda & 2.25 \\
Variance \% & 75.08 \\
\hline
\end{tabular}

Legend: Lambda - eigenvalue; Variance \% - percentage of variance explained by latent dimension, M20 L-20meter sprint from a standing start with a ball, MSLA Lslalom run with a ball, MZI-ZA L-zig-zag run with a ball.

The results of differences determined by an independent samples t-test are presented in Table 8 . The results of the independent samples t-test indicate there was a statistically significant difference between two groups of soccer players in variables sla- lom run with a ball $(\mathrm{t}-\mathrm{value}=-3.41 ; \mathrm{p}=0.01)$ and zig-zag run with a ball $(\mathrm{t}-\mathrm{value}=-3.48 ; \mathrm{p}=0.01)$. By examining arithmetic means of the two groups of subjects, it can be concluded that soccer players in the first group (players aged 11 years) achieved better results.

Table 8. Differences between two groups of soccer players in tests assessing specific agility

\begin{tabular}{ccccc}
\hline Variable & $\begin{array}{c}\text { Group 1 } \\
(\mathbf{N}=12)\end{array}$ & $\begin{array}{c}\text { Group 2 } \\
(\mathbf{N}=\mathbf{2 0})\end{array}$ & \multicolumn{2}{c}{ t-test } \\
& $\mathbf{A M} \pm \mathbf{S D}$ & $\mathbf{A M} \pm \mathbf{S D}$ & t-value & $\mathbf{p}$ \\
\hline M20 L & $4.08 \pm 0.16$ & $4.21 \pm 0.20$ & -1.94 & 0.06 \\
MSLA L & $11.10 \pm 0.56$ & $11.99 \pm 0.79$ & -3.41 & 0.01 \\
MZI-ZA L & $8.95 \pm 0.39$ & $9.63 \pm 0.60$ & -3.48 & 0.01 \\
\hline
\end{tabular}

Legend: $t$-test-independent samples t-test; $p$-level of significance, M20 L-20-meter sprint from a standing start with a ball, MSLA L-slalom run with a ball, MZI-ZA L-zigzag run with a ball.

\section{Discussion}

The primary aim of this study was to validate the measuring instruments for assessing certain motor abilities in youth soccer players. In line with this goal, values of reliability, homogeneity, sensitivity, and factor and pragmatic validity were calculated. Analyses were performed based on which the results on metric characteristics of tests assessing selected motor dimensions were obtained. Numerous previous studies (Folio \& Fewell, 2000; Ulrich, 2000) claim that values of coefficients of reliability are the most important indicator of metric characteristics. Desirable values of coefficients of reliability cannot be explicitly determined. Namely, some authors claim that coefficients of correlation must be at least 0.90 (Malacko \& Popoviæ, 2001; Salvia \& Ysseldyke, 1988) for a test to be reliable, whereas others argue that the level of correlation of 0.70 is the lower reliability limit (Folio \& Fewell, 2000; Ulrich, 2000).
Considering that analysis of homogeneity by using average inter-item correlation or factor analysis greatly depends on objectivity, i.e., variance of error, and is hard to differentiate from coefficient of objectivity, in this study homogeneity was analysed by applying analysis of variance. This type of analysis is also used by Žuvela, Maleš and Ėerkez (2009) in their study of biotic motor skills, in which the author uses lower homogeneity as indication to take the best result as the final result in a test, instead of using the mean result (heterogenous result).

In accordance with these findings and the results presented in this study, some general findings of this study may be presented. Indicators of metric characteristics for assessing sprinting speed indicate that the 20 -meter sprint from a standing start (M20M) test is the best in assessing sprinting speed. Indicators of accuracy of measurement in the analysed test are very high. According to the obtained arithmetic means it can be said that 
in the M20M test a process of motor learning has not occurred, so the same number of items in assessment of the analysed ability (homogenous test) can be recommended for future studies. The test is good in differentiating subjects regarding the object of measurement. The subjects' results do not deviate significantly from normal Gaussian distribution. Results of factor structure of motor space on a sample of youth soccer players indicate that the analysed test has the highest correlation with the object of measurement. By observing the findings of this study through parameters of pragmatic validity, the obtained results indicate there is a certain difference in the sprinting speed only in the 5-meter sprint test. The obtained differences can probably be ascribed to the difference in chronological age, but also to the knowledge on performance of standing start, which, by the authors' opinion, certainly represents an important factor for running the 5-meter distance from a standing start successfully. It can be assumed that during systematically directed kinesiological exercise boys in the older age category managed to acquire the starting skill on a higher and more quality level, which in the end has certainly led to certain differences in the 5-meter sprint.

Values of metric characteristics of tests assessing specific agility indicate that the slalom run with a ball (MSLA L) test is best in assessing the analysed ability. Correlation of results of measurement on the same object of measurement is satisfactory. Unambiguous inference in the MSLA L test is possible (homogenous test). Distribution of data in the test does not deviate significantly from normal Gaussian distribution. Out of the 3 manifest variables assessing specific agility, the slalom run with a ball test has the highest partial projection on the obtained motor factor. Also, it should be noted that in the M20L test there was a certain tendency of decrease of arithmetic mean values, which could indicate a certain motor learning process during performance of this motor task. Therefore, it would be advisable to increase the number of repetition items in future studies. On the other hand, there was a certain tendency of increase of arithmetic mean values in the zig-zag run with a ball test. This trend has probably occurred due to insufficient pause between each repetition in the test, so it would be advisable in future studies to increase the rest period between repetitions.

In line with the obtained results (pragmatic validity), it can be seen that there are certain differences between the two age categories in specific agility. As in the previously analysed difference in sprinting speed at 5-meter distance from a standing start, it is obvious that the quality of systematically selected kinesiological operators (primarily of soccer contents), has led to significant differences in specific agility. The obtained differences can be seen in almost all the applied tests.

To summarize, the values of metric characteristics indicators showed that motor tests assessing speed and specific agility had high level of reliability, good homogeneity, normal distribution of data, and acceptable factor and pragmatic validity. Results obtained in indicate that during a properly selected training process (soccer), target transformations may occur in abilities of specific agility, which are considered by most authors as being one of the most important for further development of youth soccer players. One of the most important and most frequently tested segments of anthropological status are motor abilities of athletes. Processes of selection, orientation and monitoring in the area of elite sport are inconceivable without information on athletes' motor dimensions. Without a doubt, sports can be classified into a pattern of motor activities in which these characteristics come to the fore. All motor abilities undeniably affect the results of sports achievements, but individual contributions of different motor dimensions are diverse and varying in each individual sports activity. Therefore, it is very important have a valid tool to assess motor abilities such as speed and specific agility in youth soccer. We hope that this study will provide useful information's for coaches and athletes for creating a successful training program.

\section{Acknowledgements}

There are no acknowledgements.

\section{Conflict of Interest}

The authors declare that there are no conflicts of interest.

\section{Received: 09 July 2018| Accepted: 27 August 2018| Published: 01 October 2018}

\section{References}

Buchheit, M., Mendez-Villanueva, A., Delhomel, G., Brughelli, M., \& Ahmaidi, S. (2010). Improving Repeated Sprint Ability in Young Elite Soccer Players: Repeated Shuttle Sprints Vs. Explosive Strength Training. Journal of Strength and Conditioning Research, 24(10), 2715-2722. doi. org/10.1519/JSC.0b013e3181bf0223

Chuman, K., Hoshikawa, T., \& Lida, T. (2009). Yo-Yo intermittent recovery level 2 test in pubescent soccer players with relation to maturity category. Footbal Science, 6, 1-6.

De Matos, D.G., Dantas, E.H.M., Aidar, F.J., Silva, A.C., Rodrigues, B.M., Klain, I.P, Hicker, R.C, Carneiro, A.G, Filho, M.L.M. (2013). Relationship between maturity levels and neuromuscular capacity among youth soccer players and individuals not practicing soccer. Health, 05(01), 30-34. doi. org/10.4236/health.2013.51005

Figueiredo, A.J., Gonçalves, C.E., Coelho E Silva, M.J., \& Malina, R.M. (2009). Youth soccer players, 11-14 years: maturity, size, function, skill and goal orientation. Annals of Human Biology, 36(1), 60-73. doi. org/10.1080/03014460802570584

Folio, M.R. \& Fewell, R. (2000). Peabody Developmental Motor Scales. Examiners manual. Austin-Texas: Pro-ED Inc.

Gatz, G. (2009). Complete Conditioning for Soccer. Champaign, Illinois: Human Kinetics.

Harman, E. \& Garhammer, J. (2008). Testing and evaluation. In T. Baechle \& R. Earle (Eds.), Essentials of Strength Training and Conditioning (3rd ed., pp. 237-273). Champaign, Illinois: Human Kinetics.

Lago-Peñas, C., Lago-Ballesteros, J., Dellal, A., \& Gómez, M. (2010). Game-Related Statistics that Discriminated Winning, Drawing and Losing Teams from the Spanish Soccer League. Journal of Sports Science \& Medicine, 9(2), 288-293.

Le Gall, F., Carling, C., Williams, M., \& Reilly, T. (2010). Anthropometric and fitness characteristics of international, professional and amateur male graduate soccer players from an elite youth academy. Journal of Science and Medicine in Sport, 13(1), 90-95. doi.org/10.1016/j.jsams.2008.07.004

Malacko, J. \& Popović, D. (2001). Metodologija kineziološko antropoloških istraživanja. Priština: Fakultet za fizičku kulturu Univerziteta u Prištini.

Malina, R.M., Eisenmann, J.C., Cumming, S.P., Ribeiro, B., \& Aroso, J. (2004). Maturity-associated variation in the growth and functional capacities of youth football (soccer) players 13-15 years. European Journal of Applied Physiology, 91(5-6), 555-562. doi.org/10.1007/s00421-003-0995-z

Mendez-Villanueva, A., Buchheit, M., Kuitunen, S., Douglas, A., Peltola, E., \& Bourdon, P. (2011). Age-related differences in acceleration, maximum running speed, and repeated-sprint performance in young soccer players. Journal of Sports Sciences, 29(5), 477-484. doi.org/10.1080/026404 14.2010 .536248

Miller, T. (2012). NSCA's Guide to Tests and Assessments. Champaign, Illinois: Human Kinetics.

Mujika, I., Santisteban, J., Impellizzeri, F.M., \& Castagna, C. (2009). Fitness determinants of success in men's and women's football. Journal of Sports Sciences, 27(2), 107-114. doi.org/10.1080/02640410802428071

Salvia, J. \& Ysseldyke, J. E. (1988). Assessment in special and remedial education. Houghton Mifflin Co.

Ulrich, D. (2000). Test of Gross Motor Development. Austin, TX: Pro-ed Publishers.

Váczi, M., Tollár, J., Meszler, B., Juhász, I., \& Karsai, I. (2013). Short-term high intensity plyometric training program improves strength, power and agility in male soccer players. Journal of Human Kinetics, 36, 17-26. doi. org/10.2478/hukin-2013-0002

Žuvela, F., Maleš, B., \& Čerkez, I. (2009). The influence of different learning models on the acquisition of specific athletic throwing skills. Facta Universitatis: Series Physical Education and Sport, 7(2), 197-205. 